Ann. Génét. Sél. anim., I977, 9 (3), 379-385.

\title{
Effets maternels d'un système sanguin sur le nombre d'œufs et conséquences sur la sélection dans une souche expérimentale
}

\author{
X. de LAAGE, Nicole MILlET et J. P. BOYER \\ Station de Recherches Avicoles \\ Centre de Recherches de Tours, I.N.R.A., \\ Nouzilly 37380 Monnaie
}

\begin{abstract}
Résumé
Avec des iso-immuns sérums de la souche expérimentale L 22 Nouzilly (type Leghorn à crête variable) utilisés sur des échantillons de sang de reproducteurs et de leur descendance, la totalité des 9 facteurs antigéniques simples d'un système a été identifiée.

La comparaison des génotypes sanguins avec la performance des 7 premiers mois de ponte montre que les 9 facteurs peuvent être répartis en 3 groupes - comptant chacun trois allèles selon leur association avec la ponte : positifs $(+)$ neutres $(\mathrm{N})$ ou négatifs $(-)$. Les effets, selon les facteurs, sont contrastés suivant le stade chronologique considéré pour la production.

Le facteur reçu de la mère manifeste un effet plus net et même prépondérant sur le caractère transmis par le père.

Le choix des reproducteurs, effectué sur la base d'un index de ponte, indépendamment des génotypes sanguins, montre $a$ posteriori que les animaux sélectionnés, mâles et femelles, constituent des échantillons représentatifs des facteurs paternels du troupeau initial mais, en revanche, non représentatifs des facteurs maternels. L'interprétation des résultats par un effet " chronogénétique " est suggérée.
\end{abstract}

Divers auteurs ont passé en revue l'effet de facteurs antigéniques sur les performances, de ponte notamment (par exemple Gilmour, I96o; BriLes, I96o, I969; NORDSKOG, I964).

Il nous a paru intéressant de mener parallèlement mais indépendamment l'étude d'un système sanguin et la sélection sur un index de ponte, puis d'examiner a posteriori comment la sélection révélait ou non une modification des fréquences des différents facteurs.

Plutôt que de mettre en évidence des différences de ponte liées à des différences antigéniques connues a priori, cette démarche a contrario permettrait de montrer l'existence éventuelle d'associations entre production d'œufs et génotype sanguin. 


\section{Matériel et méthodes}

Nous avons utilisé ioo femelles de la souche L 22 (expérimentale, de type Leghorn avec ségrégations pour le type de crête) s'immunisant réciproquement par couple de sœurs selon la voie intramusculaire; puis 30 autres, parentes ou non, dont les échantillons de sang furent analysés avec les sérums produits par les précédentes. Quinze nouveaux couples de donneurs-receveurs, chacun d'eux ayant réagi avec les mêmes anticorps, furent ainsi constitués. Il en fut de même pour attribuer un nouveau donneur aux animaux dont on n'avait obtenu aucune réponse immunologique. Ce petit cheptel a servi ensuite pour l'étude des sérums récoltés et pour de nombreux tests de pureté. On a retenu 15 réactifs spécifiques et 20 autres sérums à définir, qui ont été utilisés pour l'analyse du sang de reproducteurs L 22 ( 36 coqs et 87 poules) et de leur descendance (environ I 600 enfants, âgés de ro semaines, issus de 3 lots d'éclosion).

La mise en évidence des facteurs sanguins de cette souche et des allélismes, résultant de la ségrégation des caractères parentaux, a permis de grouper 9 sérums détectant la totalité des allèles d'un même locus : chaque échantillon de sang réagissait avec un ou deux de ces réactifs et l'analyse des ascendants père et mère confirmait la possibilité d'une homozygotie ou d'une hétérozygotie. Dans le troupeau de pondeuses suivi jusqu'à la $30^{\mathrm{e}}$ semaine de ponte après le premier $œ u f$, il a été ainsi possible de constituer un lot de 530 poules pour lesquelles étaient bien identifiées les provenances paternelle et maternelle de chaque facteur.

Pour l'ensemble du troupeau, la ponte a été répartie en trois périodes successives de ro semaines et, pour chaque période, les animaux ont été catégorisés selon la valeur de leur écart réduit, les classes et leurs limites étant indiquées par la séquence $(\mathrm{D},-7 / 6, \mathrm{C},-\mathrm{I} / 6, \mathrm{~B},+5 / 6, \mathrm{~A})$. Un pourcentage important de poules peu précoces se sont trouvées classées ipso facto en catégorie $\mathrm{D}$ pour la première période de ponte.

Le croisement des données ponte et facteurs sanguins a ainsi pu être établi. Pour chaque facteur, la comparaison $(\mathrm{A}+\mathrm{B})$ vs $(\mathrm{C}+\mathrm{D})$ a été testée. Puis la comparaison $(A+D)$ vs $(B+C)$ dans les cas non significatifs. Puis en fin, pour un facteur douteux, les comparaisons $(A)$ vs $(B+C+D)$ et $(A+B+C)$ vs (D).

Enfin, après le choix des reproducteurs sur un index de production d'œufs, on a testé la représentativité des échantillons sélectionnés, mâles et femelles, par rapport au troupeau initial, puis par rapport aux facteurs d'origine paternelle et maternelle.

Les coqs ayant subi une première sélection sur la forme de la crête à l'âge de 3 mois (88 gardés sur 752) il a été tenu compte de ce fait dans l'analyse.

\section{Résultats}

Dans le cas de la souche $L$ 22, le système sanguin étudié est clos avec 9 allèles. Bien qu'on n'ait pas eu la possibilité matérielle d'identifier les sérums correspondants à ceux de la classification internationale, plusieurs indices désignent le groupe B comme vraisemblable : polymorphisme important, bonne persistance des anticorps, rapidité des agglutinations. Le système concerné ici est donc simple- 
ment identifié selon l'ordre d'exploitation des réactifs utilisés ou les numéros des poules les ayant fourni : 2, 6, 8, 9, II, I2, I4, 37, 82 .

Le tableau $\mathrm{I}$ indique, pour chaque période de ponte, le nombre de poules de chaque catégorie $\mathrm{A}, \mathrm{B}, \mathrm{C}, \mathrm{D}$, marquées par le facteur indiqué en abscisse.

\section{TABLEAU I}

Effectif des pondeuses, par période et par catégorie, possédant l'antigène porté en abcisse Numbers of hens, by periods and classes, carrying each antigen

\begin{tabular}{|c|c|c|c|c|c|c|c|c|c|c|c|}
\hline \multirow{2}{*}{ Période } & \multirow{2}{*}{ Catégorie } & \multicolumn{9}{|c|}{ antigène } & \\
\hline & & 2 & 6 & 8 & 9 & I I & 12 & I 4 & 37 & 82 & \\
\hline I & $\begin{array}{l}\mathrm{A} \\
\mathrm{B} \\
\mathrm{C} \\
\mathrm{D}\end{array}$ & $\begin{array}{r}22 \\
29 \\
23 \\
26 \\
100\end{array}$ & $\begin{array}{r}35 \\
91 \\
98 \\
\text { I I I } \\
335\end{array}$ & $\begin{array}{r}\text { I6 } \\
28 \\
3 \mathrm{I} \\
29 \\
\text { I04 }\end{array}$ & $\begin{array}{r}33 \\
50 \\
39 \\
32 \\
\mathbf{5} 54\end{array}$ & $\begin{array}{l}\text { I } 4 \\
32 \\
\text { I } 4 \\
26 \\
86\end{array}$ & $\begin{array}{r}26 \\
63 \\
66 \\
72 \\
227\end{array}$ & $\begin{array}{r}9 \\
\text { I } 4 \\
\text { I } 6 \\
\text { I } 4 \\
53\end{array}$ & $\begin{array}{r}31 \\
38 \\
32 \\
59 \\
160\end{array}$ & $\begin{array}{r}I \\
\text { IO } \\
\text { II } \\
8 \\
30\end{array}$ & $\begin{array}{r}\text { I } 87 \\
355 \\
330 \\
377 \\
\text { I } 249\end{array}$ \\
\hline II & $\begin{array}{l}\mathrm{A} \\
\mathrm{B} \\
\mathrm{C} \\
\mathrm{D}\end{array}$ & $\begin{array}{r}28 \\
39 \\
25 \\
10 \\
102\end{array}$ & $\begin{array}{r}47 \\
107 \\
\text { I } 28 \\
48 \\
330\end{array}$ & $\begin{array}{l}\text { I4 } \\
39 \\
33 \\
\text { I } 3 \\
99\end{array}$ & $\begin{array}{r}29 \\
47 \\
55 \\
20 \\
\text { I } 51\end{array}$ & $\begin{array}{l}20 \\
35 \\
\text { I } 8 \\
12 \\
85\end{array}$ & $\begin{array}{r}31 \\
68 \\
85 \\
38 \\
222\end{array}$ & $\begin{array}{r}\text { I } 2 \\
2 \mathrm{I} \\
\text { I } 5 \\
3 \\
5 \mathrm{I}\end{array}$ & $\begin{array}{r}32 \\
43 \\
51 \\
25 \\
151\end{array}$ & $\begin{array}{r}5 \\
12 \\
7 \\
4 \\
28\end{array}$ & $\begin{array}{r}218 \\
411 \\
417 \\
\text { I } 73 \\
1219\end{array}$ \\
\hline III & $\begin{array}{l}\text { A } \\
\text { B } \\
\text { C } \\
\text { D }\end{array}$ & $\begin{array}{r}24 \\
35 \\
27 \\
8 \\
94\end{array}$ & $\begin{array}{r}48 \\
98 \\
\text { I } 13 \\
58 \\
317\end{array}$ & $\begin{array}{l}20 \\
22 \\
30 \\
13 \\
85\end{array}$ & $\begin{array}{r}26 \\
45 \\
54 \\
\text { I6 } \\
\text { I } 4 \text { I }\end{array}$ & $\begin{array}{r}19 \\
26 \\
25 \\
5 \\
75\end{array}$ & $\begin{array}{r}27 \\
76 \\
69 \\
4 \mathrm{I} \\
2 \mathrm{I} 3\end{array}$ & $\begin{array}{r}\text { I } 2 \\
19 \\
\text { I6 } \\
2 \\
49\end{array}$ & $\begin{array}{r}29 \\
4 \mathrm{I} \\
42 \\
28 \\
\mathrm{I} 40\end{array}$ & $\begin{array}{r}5 \\
10 \\
6 \\
4 \\
25\end{array}$ & $\begin{array}{r}210 \\
372 \\
382 \\
175 \\
\text { I } 139\end{array}$ \\
\hline
\end{tabular}

Le tableau 2 indique le résultat du test $\chi^{2}$ appliqué aux comparaisons indiquées précédemment. Il en ressort que les 9 facteurs antigéniques peuvent être regroupés en trois classes, selon leur incidence sur la ponte :

facteurs positifs $(2$, II, I 4$)=+$

facteurs neutres $(8,9,82)=\mathrm{N}$

facteurs négatifs $(6,12,37)=-$ (noter la tendance disruptive de l'antigène 37$)$.

Dans ce qui suit, on ne considère que ces trois classes $(+, \mathrm{N}, \rightarrow)$ étant donné que l'échantillon des reproducteurs, assez peu important, conduirait à des fréquences théoriques insuffisantes pour valider un test détaillé.

Le tableau 3 est établi avec seulement trois catégories définies par les troncatures d'écarts-réduits indiqués dans la séquence (III, $-2 / 5$, II, $+\mathrm{I} / 3$, I). Il contient la proportion de ces trois catégories parmi les pondeuses possédant des facteurs antigéniques à effet " + ), " $\mathrm{N}$ " ou " - " et suivant l'origine paternelle ou maternelle de ces facteurs. 


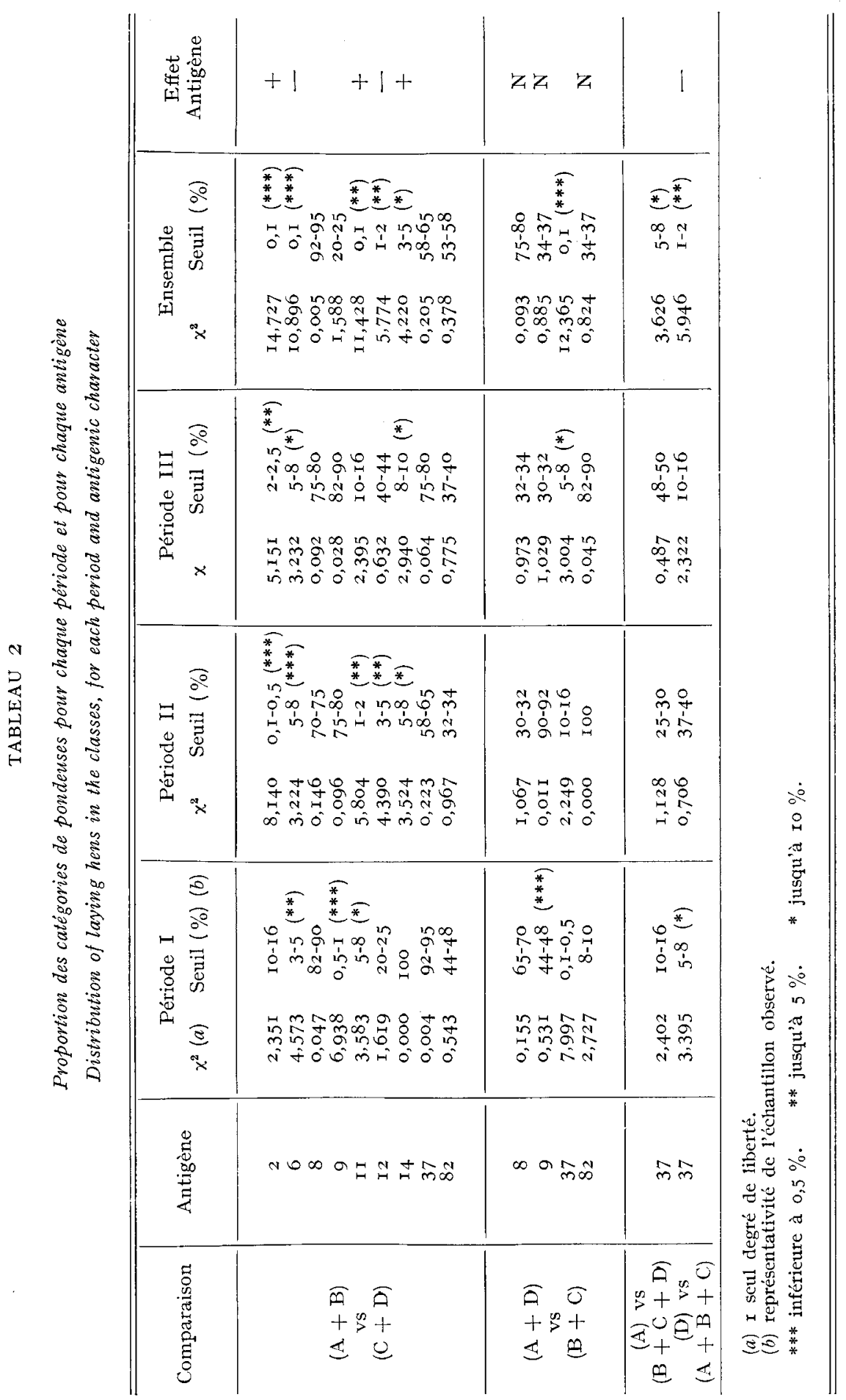




\section{TABLEAU 3}

Proportion des différentes catégories de pondeuses selon le type et la provenance des facteurs antigéniques

(Proportion of the classes for egg production according to type and origin of antigenic factors)

\begin{tabular}{|c|c|c|c|}
\hline \multirow{2}{*}{$\begin{array}{l}\text { Type et provenance } \\
\text { de l'antigène }\end{array}$} & \multicolumn{3}{|c|}{ Catégorie des pondeuses } \\
\hline & I & II & III \\
\hline $\begin{array}{l}\text { + maternel. . . . } \\
+ \text { paternel . . }\end{array}$ & $\begin{array}{l}55, \mathrm{I} \\
42,5\end{array}$ & $\begin{array}{l}25,5 \\
30,0\end{array}$ & $\begin{array}{l}19,4 \\
27,5\end{array}$ \\
\hline $\begin{array}{l}\mathrm{N} \text { maternel. . . . . } \\
\mathrm{N} \text { paternel . . }\end{array}$ & $\begin{array}{l}33,3 \\
4 I, 8\end{array}$ & $\begin{array}{l}33,3 \\
24,0\end{array}$ & $\begin{array}{l}33,3 \\
34,2\end{array}$ \\
\hline $\begin{array}{llll}\text { —maternel. } . ~ & . & . & . \\
\text {-- paternel . . } & . & . & \text {. }\end{array}$ & $\begin{array}{l}29,6 \\
31,1\end{array}$ & $\begin{array}{l}25,2 \\
27,2\end{array}$ & $\begin{array}{l}45, \mathrm{I} \\
4 \mathrm{I}, 7\end{array}$ \\
\hline
\end{tabular}

La différence de proportion des catégories I, II et III est nettement plus importante pour les facteurs maternels $(+,-)$ que pour les facteurs d'origine paternelle. Un $\chi^{2}$ de contingence établi pour les premiers (proportion de facteurs + , $\mathrm{N}$ et - parmi les catégories I, II et III) atteint la valeur 29,9 pour $4 \mathrm{~d}$. 1 . $(\mathrm{P}<0, \mathrm{ooI})$ alors que pour les facteurs d'origine paternelle la valeur du $\chi^{2}$ analogue est 9,9 $(\mathrm{P} \simeq 0,05)$.

\section{TABLEAU 4}

Effectif, selon le génotype sanguin, des coqs et poules avant et après sélection pour le nombre d'ceufs

(Numbers, for each blood type, of cocks and hens before and after selection for egg number)

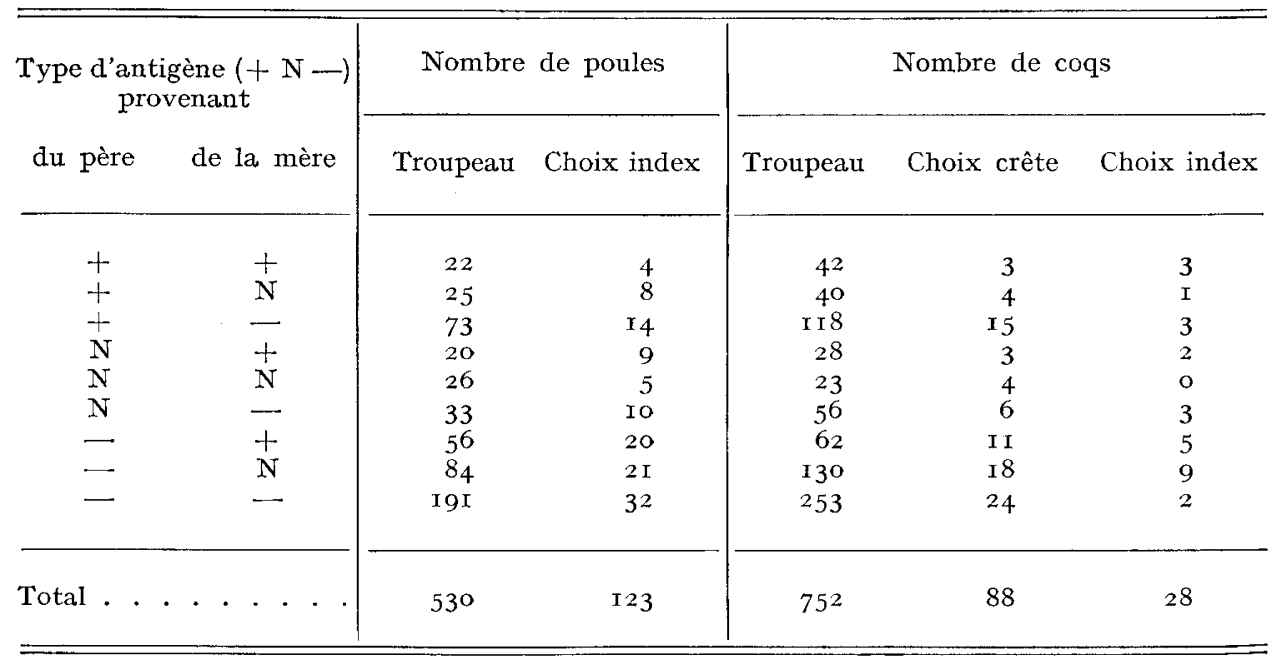


Enfin les tableaux IV et $\mathrm{V}$ montrent que les échantillons de reproducteurs, choisis sur la production d'œufs, sont représentatifs de la distribution d'origine des facteurs paternels mais, en revanche, non représentatifs de celle des facteurs maternels $\left(P^{2}<0,05\right)$. En somme, la sélection pour la ponte a modifié la répartition des allèles maternels, au profit des positifs, au détriment des autres, sans modifier par ailleurs celle des allèles paternels.

Il en va de même pour les deux sexes. On notera en passant que les gènes affectant la forme de la crête (dans le cas présent les caractères simple/rosacé et hérissé/lisse) sont indépendants du locus sanguin étudié.

TABLEAU 5

Représentativité des reproducteurs choisis sur index pour la ponte

Breeding birds chosen on a laying index as representative of the flock for blood types

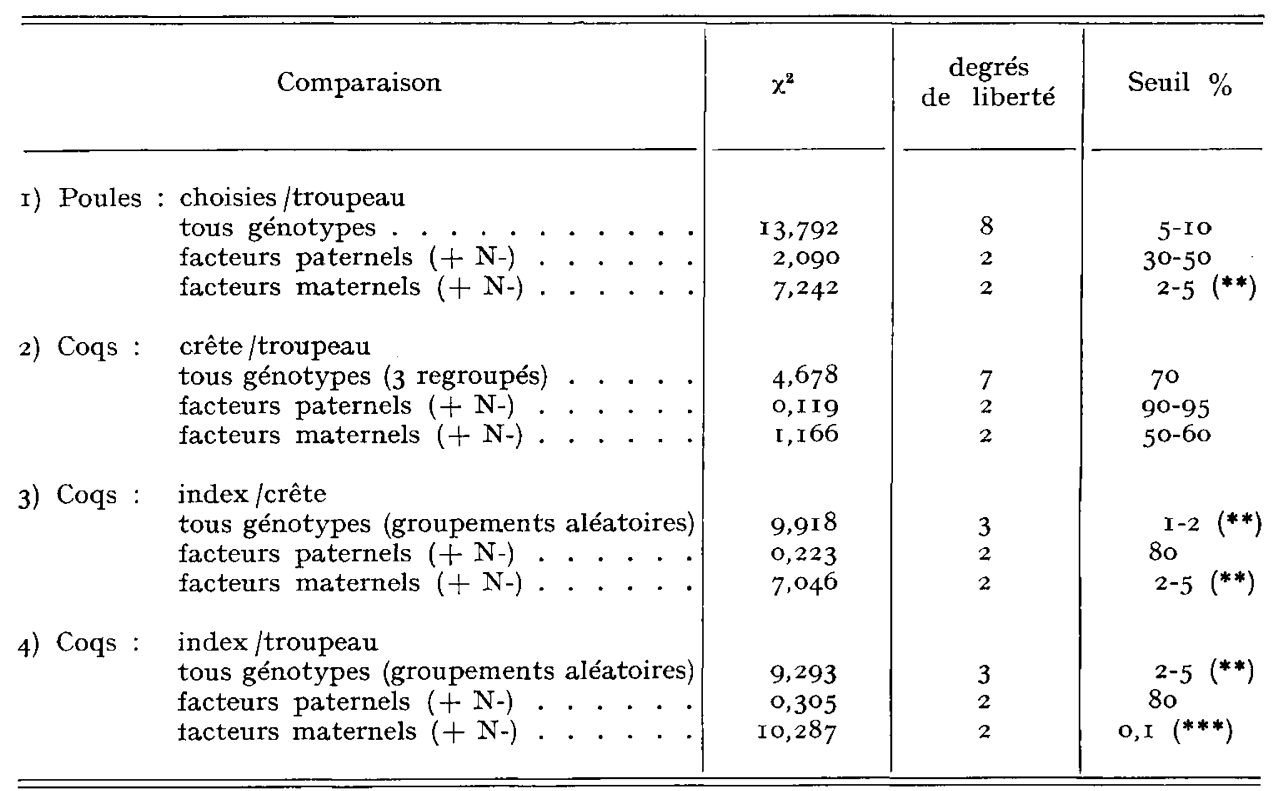

\section{Discussion}

Deux effets chronologiques semblent intéressants à considérer. D'une part, même si l'effet total permet de distinguer trois types d'allèles, ceux ayant une influence sur la ponte se manifestent pour certains d'une manière contrastée selon la période considérée. Ainsi l'allèle 2, très puissant en périodes II et III, n'a aucun effet en début de ponte. Inversement l'allèle 9, neutre dans l'ensemble, montre un effet pourtant très marqué en période I, etc.

Ces résultats suggèrent une interaction génotype-milieu, un environnement particulier caractérisant chaque période; on peut penser à un effet "chronogénétique ", l'information génétique restant silencieuse ou active selon chaque allèle 
pendant des périodes de ponte relativement précisées ici, par tranche de Io semaines à compter du premier œuf.

D'autre part, la relation des facteurs antigéniques de provenance paternelle est, dans tous les cas, non significative. Tout se passe comme si l'information génétique paternelle était non ou faiblement exprimée. Ceci suggère une forme particulière d'hérédité maternelle et conduit à se poser la question : les jeux sont-ils faits avant l'amphimixie?

C'est une manière d'évoquer à nouveau l'hypothèse d'un effet chronogénétique précocissime. On notera que dans un tel modèle, les coqs transmettent à leurs filles un facteur paternel qui reste sans effet sur la ponte, mais qui sera transmis à leurs petites filles, par la voie femelle, comme facteur d'origine maternelle, cette fois réactivé.

Dans le domaine appliqué il apparaît donc que le choix des reproducteurs sur un index ponte ne conduit pas à une sélection sur les allèles paternels. Au contraire, la modification de la répartition des allèles maternels est importante chez les femelles et chez les mâles. Or le sélectionneur a une certaine latitude dans sa décision finale et l'observation a montré dans l'étude présente qu'elle aurait pu dans bien des cas mieux s'exprimer, au profit des facteurs sanguins. Appliquer à ce critère cette marge d'appréciation, lourde de conséquence lorsqu'il s'agit du choix des mâles, situerait cette ultime décision à son niveau, celui de la génétique.

Reçu pour publication en juillet 1977

\begin{abstract}
Summary
Maternal effects of a blood system upon the number of eggs, the results for the selection in an experimental strain
\end{abstract}

An antigaic system of a blood group-presumably B-closed with 9 factors is identified in an experimental type Leghorn strain. These factors can be divided into 3 classes (positive, neutral, negative)-each one of 3 factors, according to their association with egg number laid. Their effects are compared in relation to the period of laying considered. 36 sires and 87 dams were analysed, and their offspring too (about 1600 ). The factor received from the dam shows a better association with the production than the sire's one.

The selection of the males, on the basis of a production index, independently of blood. group, shows a posteriori that the selected animals constitute a representative sample of the paternal factors but a non-representative sample of the maternal factors.

A "chronogenetic" interpretation of the results is suggested.

\title{
Références bibliographiques
}

Briles W. E., I960. Blood groups in chickens, their nature and utilization. World's Poult. Sci. J. I6, 223-242.

Gilmour D. G., 1960. Blood groups in chickens. Brit. Poult. Sci. r, 75-10o.

Gilmour D. G., I969. Blood groups research in chickens. Agric. Sci. Review 7, 13-22.

Nordskog A. W., I964. Poultry immunogenetics. World's Poult. Sci. J. 20, I83-192. 